\title{
Colored Pencil Drawing Method Based on HSV Color Space
}

\author{
Feiyue Chen ${ }^{1, a^{*}}$, Congli Lu, ${ }^{1, b}$ and Jingang Liu ${ }^{1,2, c}$ \\ ${ }^{1}$ College of Information Engineering, Capital Normal University, Beijing, China; \\ ${ }^{2}$ Institute of Computing Technology Chinese Academy of Sciences, Beijing, China; \\ agoodcfy@126.com, ${ }^{\mathrm{b}}$ conglilu@outlook.com, 'liujg2000@163.com
}

Keywords: Linear integral convolution (LIC); LAB space color differentiation image; Arctangent hue differentiation model (arc tan); HSV color space

\begin{abstract}
In this paper, we propose a new colored pencil drawing generation framework which aims at simulating pencil drawing by computer at this stage. It can generate a colored pencil drawing from a 2D image automatically. The framework is built based on a generation of gray pencil drawing. Firstly, in order to draw lifelike grayscale texture we put forward an adaptive hue differentiation model to match different image automatically according to the gray histogram statistics for achieving a dark, grey and bright layered effect. Secondly, in order to generate color texture we assign grayscale texture value to channel V in HSV color space. After that we get the final result by assigning grayscale texture value to channel $S$ according to the value of channel V. Thirdly, for the first time we replace part of texture image with part of LAB space aberration image in order to get outer contour. From the final effect, our algorithm have three advantages over other algorithms, one is a better dark, grey and bright hue differentiation effect and a better contrast, the second point is that the outer contour is more coherent and clear. Thirdly, the hue change of colored texture is grey, color and white, which includes both light and shade contrast and color change. Such colored texture is closer to a real aesthetic feeling.
\end{abstract}

\section{Introduction}

Pencil drawing is detail rich and expressive, diverse style and form and etc. Colored pencil drawing as an extension of pencil drawing is appreciated by more and more people. In the early days, some approaches to colored pencil drawing are to imitate the watercolor effect intentionally. Compared to watercolor painting, colored pencil drawing can be able to better show delicate texture of objects. With the development of technology, a variety of electronic equipment's are constantly emerging, especially for the mobile terminal which speeds up commercialized progress of colored pencil drawing. We have to say that colored pencil drawing is more and more capturing people's attention.

The key for simulating colored pencil drawing is how to draw color texture. Early approaches are mostly limited to gray pencil drawing generation ideas such as ideas based on physical laws, paper structure, pigment properties and etc. But these algorithms request higher complexity. In recent years, some people propose an idea to generate color texture by means of color customization. This is to say that we need to divide image into different zones firstly and secondly decide several optimal color to produce a multi-level image for each region. However a subjective man-made customization leads to a deviation from original image hue. Some others put forward to converting RGB color space to others such as YUV or HIS for generating colored pencil drawing. After that we can generate color texture by changing hue, brightness or other attributes based on different color spaces with specific parameter properties. An advantage of this approach is that it can be better able to generate color texture based on the original image hue. In this paper, a new algorithm is proposed following the color space transformation theory. We choose the HSV color space because of the change of channel S and V which can satisfy the demand for color texture's hue variation.

In this paper, we put forward a new colored pencil drawing generation framework based on the gray pencil drawing. It is divided into two parts, the first part is gray pencil drawing generation, and the second is about colored pencil drawing. We put the gray pencil part in the framework as a foundation for generating colored pencil drawing. In order to make the final colored pencil drawing effect better, the first thing we need to do is a good job of simulating gray pencil drawing. So we make several new 
attempts to gray texture, outer contour extraction and dark, grey and bright hue differentiation. According to a hue differentiation thought proposed by Lu [1], the arctangent hue differentiation model is hereby being proposed to reflect hue layered effect. Its core idea, based on dark, grey and bright layers' pixel ratio, is adjusting pixel grey value to achieve layering tones. Instead of the traditional gradient image approach, a new method proposed by us is used for outer outline extraction through combining part of LAB space color difference image and texture image. The second part of our framework mainly expounds color texture generation process. The most important difference between gray pencil drawing and colored is a difference between color texture and gray texture. The hue change of gray pencil drawing is dark, grey and bright. While color texture's hue change is a combination of light and shade and color change. Based on this, we propose a theory to generate color texture by assigning gray texture's hue change rule to channel S and V in HSV color space. This approach has achieved good effect as we expected. The final result achieves texture's light and shade contrast and color change.

\section{Previous Work}

Based on the gray pencil drawing simulation, many people want to get more rich artistic effect. For this purpose, they add color information to gray pencil drawing. So far many colored pencil drawing algorithms have been proposed. Color texture is the key to simulating colored pencil drawing. In the literature [2], Color-mixture is pointed out to generate color texture, two methods are put forward in it, one is glazing which daubs a layer of color to the previous color layer again and again for achieving multilayer color mixing effect, and another is color line juxtaposition which can enhance texture contrast. Takagi [3] presents a simulation method based on physical laws to colored pencil drawing which embodies paper microstructure, pigment redistribution and erasure effect. In the literature [3-4], although a colored pencil simulation thought is given, there is no specific stroke generation process. Yams Moto [5] proposes a method called color customization which divides source image into different areas, decides two kinds of optimal color for each area and calculates color density to create two layers of images. After that the LIC method is used to produce texture map. Finally, they fuse two layers of images to get the final result by using the Kubelka-Munk optical fusion model [6]. However this scheme is still flawed because of not matching the original image hue greatly. Kang [7] put forward another approach to customize color which samples color from input image to achieve a more accurate color customization. Xie [8] generates a colored pencil drawing based on grayscale and local color diffusion theory. Xie [9] converts RGB color space to HIS and get result by adding color information of channel $\mathrm{H}$ and $\mathrm{S}$ to gray pencil. But they don't provide specific practicing process. Lu motions in [1] that they complete a transformation process from gray pencil drawing to color through simply substituting gray pencil drawing with original parameter Y in YUV color space, its nature is only to change the channel which express brightness. The texture effect were a special gray effect inherently. So the result image with characteristics of gray pencil can't really reflect features of colored pencil. Way [10] proposes a colored pencil style framework which includes paper modeling, pigment particle distribution, silhouette rendering and global stroke direction estimation. The main characteristics of the framework is shorter rendering time and a unique drawing style.

\section{Colored Pencil Drawing Method Based on HSV Color Space}

Based on the previous two sections in this paper, this part will show you four aspects to expound specifically the pencil drawing generation framework including the generation of grayscale texture, the arctangent hue differentiation model (arc tan), outer contour extraction and the generation of colored texture. In this framework, grayscale texture generation and contrast adjustment are the foundation, the arctangent hue differentiation model (arc tan) is an important link and the generation of colored texture is the key.

Fig. 1 is the whole generation flow chart of pencil drawing. Specific drawing process can briefly be showed as follows:

1) Generate noise texture from noise image by matching grayscale image. 
2) Generate texture image through putting the result from 1) and a vector field assigned by you for the linear integral convolution (LIC).

3) Weight the arctangent hue differentiation model (arc tan) to the result from 2).

4) Convert RGB color space to LAB and complete a calculation to get aberration image.

5) Get the final gray pencil drawing through combining results from 3 ) and 4).

6) Convert RGB color space to HSV.

7) Assign the result from 3) to channel $\mathrm{V}$ of the result from 6).

8) Assign the result from 3) to channel $\mathrm{S}$ of the result from 7) according to channel $\mathrm{V}$.

9) Get the final colored pencil drawing through combining results from 8) and 4).

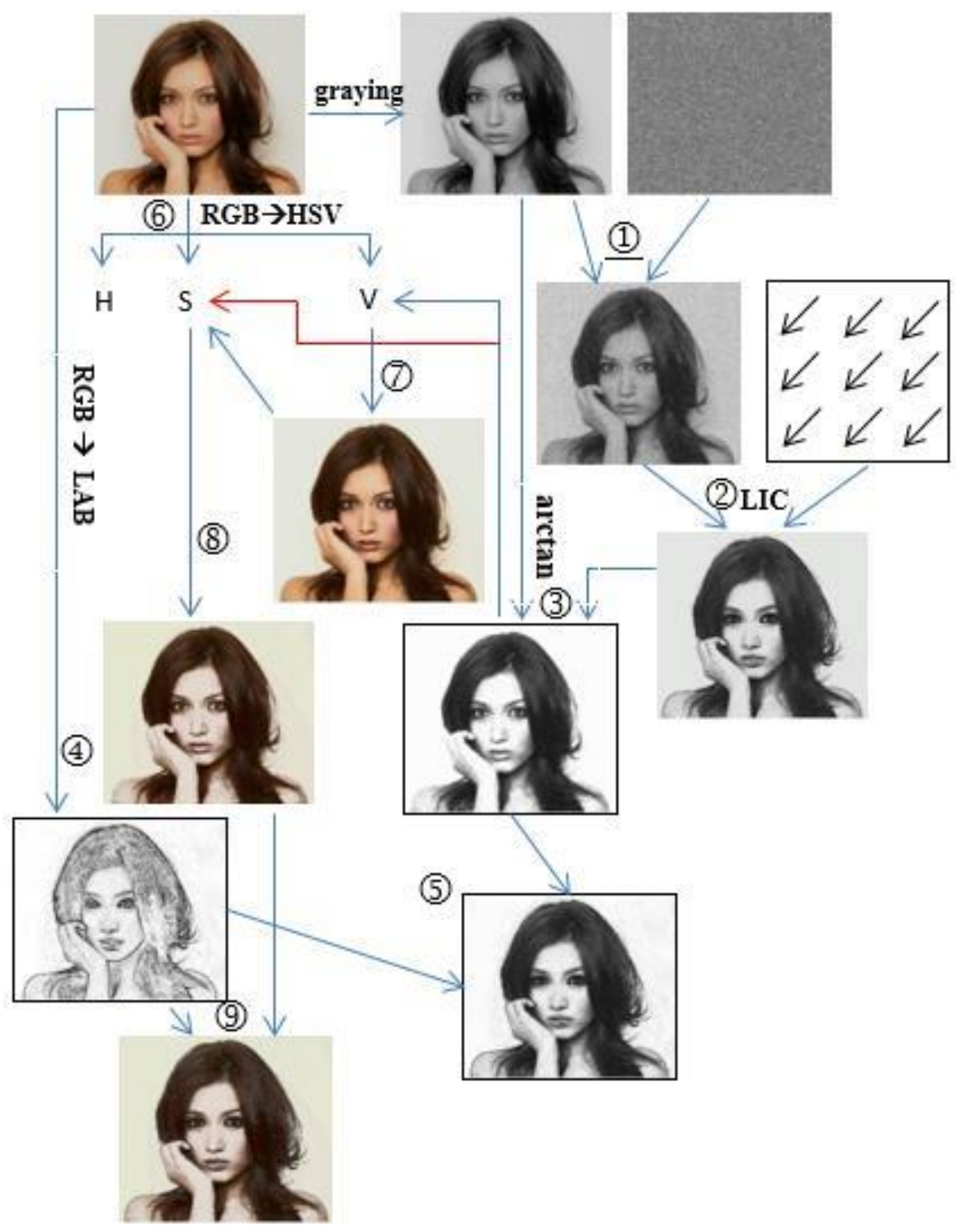

Figure 1. Pencil drawing generation framework in this paper

Arctangent Hue Differentiation Model. Lu [1] pointed out that the real pencil drawing has a more intuitive dark, gray and bright hue delamination character. So based on that, we need to seek for a hue differentiation model to sketch hue for a layered effect. In this paper, we put forward a new model also called the arctangent hue differentiation model ( $\arctan$ ) to solve this problem. The model is made up of two arc tangent function got by translation and weighted transformation. The model formula is as follows: 


$$
\begin{aligned}
& W=\frac{k}{10 \pi}\left(\arctan \frac{x-T_{1}}{2^{10 * p}}+\arctan \frac{x-T_{2}}{2^{10 *}}\right)+1, \\
& 0<T_{1}<T_{2}<255,0 \leq k<10,0 \leq p \leq 1
\end{aligned}
$$

This model is a self-adapting hue differentiation model, which could conduct parameter adjustment accordingly in accordance to different images. Its role is to implement a dark, gray and bright hierarchical contrast stretching effect on the whole. There are four parameters in this model including $T_{1}, T_{2}, \mathrm{k}, \mathrm{p}$, which $T_{1}$ and $T_{2}$ are the artificially specified threshold, which divides a grayscale image within 0 255 into three image layers. The $\mathrm{k}$ value in the model will decide $\mathrm{W}$ value which shall be within $[1-0.2 * \mathrm{k}$, $1+0.2 * \mathrm{k}]$. With parameter $\mathrm{p}$ as a scale coefficient, which means the proportion between the pixel and the total pixels within $0 \sim T_{1}$. It also means the one which has a bigger proportion between the pixel and the total pixels within $T_{2} \sim 255$. This is to explain why there are more areas to have a relatively dark or bright color if the proportion that the pixel is occupied is bigger within $0 \sim T_{1}$ or $T_{2} \sim 255$ ranges. Hence, more elaborate hue changes are needed to work on texture drawing and the weighted value added to the texture value shall not be too big to cause losses of important texture features, so that a contrast stretch effect could be achieved on the whole.

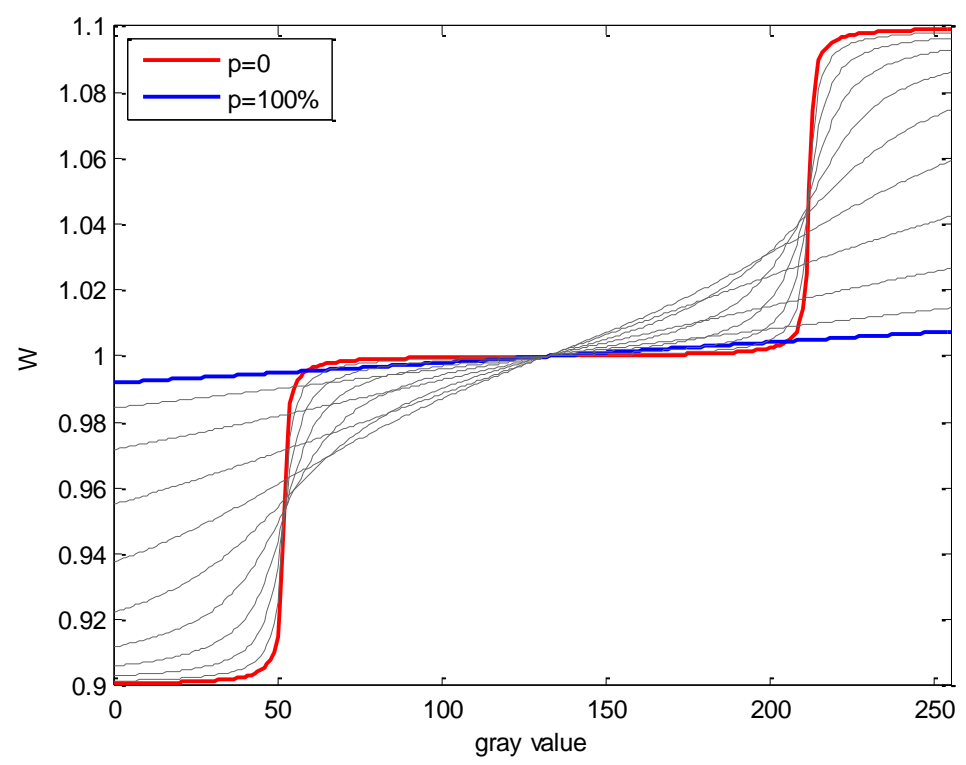

Figure 2. $w$ Changing trend with the parameter $p$

Fig. 2 shows the trend of $\mathrm{W}$ along with parameter $\mathrm{p}$ change. In the picture the red curve and the blue one respectively represent $\mathrm{W}$ value when $\mathrm{p}=0$ or $\mathrm{p}=100 \%$. It is an evident thing that the red curve can make a fully stretch for dark and bright parts of an image. However, parameters $T_{1}, T_{2}, \mathrm{k}$ in the formula need to be assigned manually. So the model adaptive ability is not strong as expected. In order to solve this problem, we consider using parameter $\mathrm{p}$ to limit parameter $T_{1}, T_{2}, \mathrm{k}$. The model formula is improved as follows:

$$
W=\frac{1-p}{\pi}\left(\arctan \frac{x-(60-15 * p)}{2^{10 * p}}+\arctan \frac{x-(205+15 * p)}{2^{10 * p}}\right)+1,0 \leq p \leq 1
$$

We know that there is not yet a clear limit to parameter $T_{1}$ and $T_{2}$. So we can specify a range for them .The $T_{1}$ value is limited to 45 60 and $T_{2}$ value is limited to 205 220. We expect the value of $T_{1}$ to enlarge and the value of $T_{2}$ to decrease when $\mathrm{p}$ value increases. With the proportion of both parameters increasing, we should weaken stretch to preserve more texture details. We expect $T_{1}$ value to decrease and $T_{2}$ value to enlarge when the value of $\mathrm{p}$ decreases. The reason is that we should appropriately increase stretch in order to enhance contrast when pixel proportion of both ends of image reduces. So the formula for limiting $T_{1}$ and $T_{2}$ is: 


$$
\left\{\begin{array}{l}
T_{1}=60-15 * p \\
T_{2}=205+15 * p
\end{array}\right.
$$

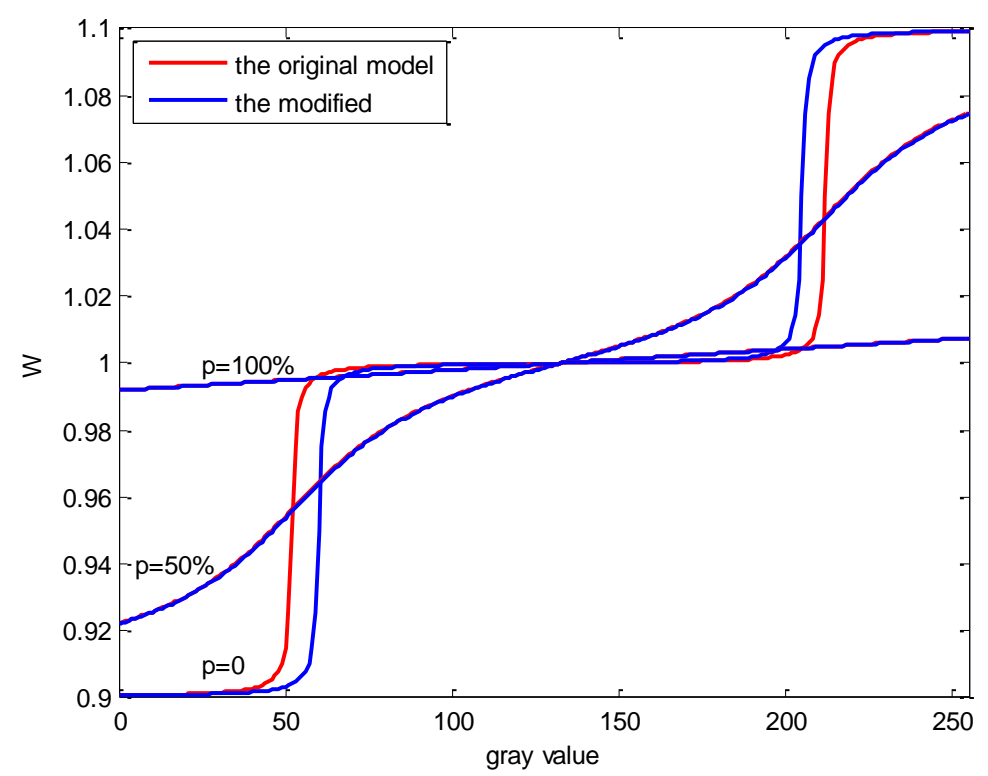

Figure 3. The modified model effect matching the original model

Fig. 3 shows an improved model effect matching the original model. The red curve denotes the original model and the blue one represents the improved model. In Fig. 3, the red curve almost matches the blue one when $\mathrm{p}$ take different values, which verifies an adaptive property of parameter $T_{1}$ and $T_{2}$.

We still use the change tendency of parameter $\mathrm{p}$ to express parameter $\mathrm{k}$, the formula is as follows:

$$
\mathrm{k}=10 *(1-\mathrm{p})
$$

The formula shows that factor $\mathrm{k}$ becomes smaller when factor $\mathrm{p}$ increases. At this time because the grayscale range on the whole is relatively narrow, we only need a little stretch for more texture details. Similarly, $\mathrm{k}$ value increases when $\mathrm{p}$ value decreases, the grayscale range on the whole enlarges and we can increase stretch appropriately. Fig. 4 shows an image with completely adaptive model $\mathrm{W}$ that has stronger contrast and more realistic effect.

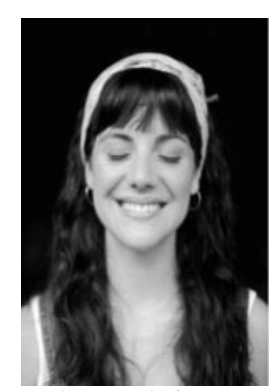

(a)Grayscale image

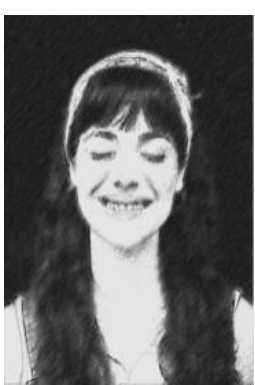

(b) Grayscale image without $W$

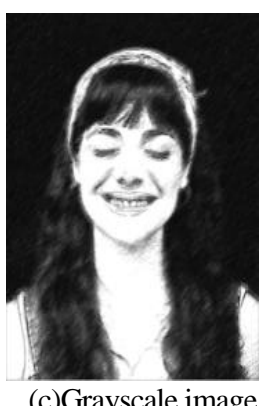

(c)Grayscale image weighted by $W$

Figure 4. Pencil drawing weighted W 
LAB Space Color Differentiation Drawing. Take a careful observation to the pencil drawing; it is clear that the texture occupies the most proportion of the entire pencil drawing, with outline only being indicated by the limits from textures with different hues. Imagine if it turns to LAB space and color differentiation drawing is adopted to replace outline, a perfect effect is expected to be obtained.

Different from RGB and CMYK color space, LAB color is designed to get close to human's vision. It focuses on the perceptual uniformity, with its L weight perfectly matching with human's perception for brightness. Hence, LAB color differentiation is more precise to perceive the tiny color changes. The calculation for LAB space color differentiation adopts Euclidean distance- $\triangle \mathrm{E}$. It is assumed to have two points existed in space as $\left(L_{1}, a_{1}, b_{1}\right)$ and $\left(L_{2}, a_{2}, b_{2}\right)$ and the method mentioned in this paper with $\Delta \mathrm{E}$ as the basis, the absolute value, generated from the brightness differentiation value between the two points, will be adopted as the weighting coefficient. This is based on the fact that human has a more sensitive perception on brightness than color. That is,

$$
\Delta \mathrm{E}=\left|L_{2}-L_{1}\right| \sqrt{\left(L_{2}-L_{1}\right)^{2}+\left(a_{2}-a_{1}\right)^{2}+\left(b_{2}-b_{1}\right)^{2}}
$$

Firstly, the color differentiation between pixels and adjacent eight points need to be calculated, and then the maximum value is adopted and kept as the final results. The automatic contrast adjustment shall be conducted in the end to make the outline more distinct.

In Fig. 5, the outline extraction results have been adopted as examples for different filters. It is obvious that Canny algorithm has a clear impact from noises, with outline occurred by inconsistency. While the segment filter has improved a lot compared with Canny algorithm. In figure (c), however, the outline is formed by short lines which are not shown naturally and smoothly. This problem can be resolved by curve filter. Compared to (c), (d) is showing more natural and smooth, but it is clear from observation that (d) has an unclear outline; (e) adopts LAB space color differentiation and automatic contrast adjustment to acquire the results, leading to a better smoothness and clarity.

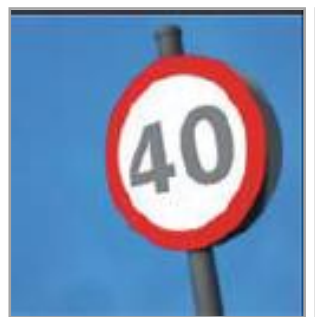

(a) Original image

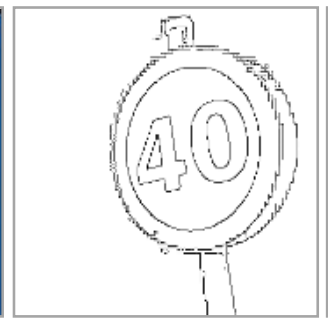

(b) Effect drawing by

Canny algorithm

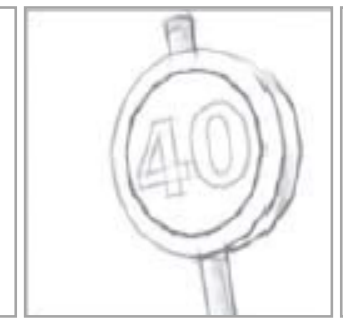

(c) Effect drawing for $\mathrm{Lu}[9]$

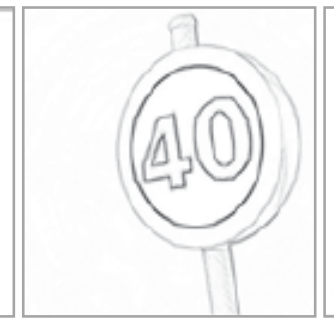

(d) Effect drawing for Lin[11]

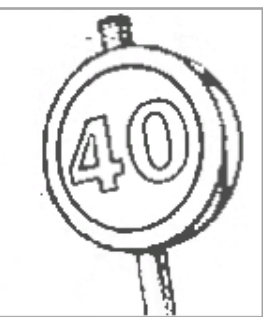

(e) LAB space color differentiation drawing

Figure 5. Outline extraction contrast with different filter as the basis

\section{The Generation of Colored Texture}

Change of Channel S and V in HSV Color Space. The hue change of colored texture should be not only light and shade but also color that can better approach the real. We find that we can achieve such a effect through changing channel S and V in HSV color space. From the Fig. 6 effect, on the horizontal direction, factor $\mathrm{V}$ changes from 0 to 255 , factor $\mathrm{S}$ stays the same and the hue variation is from dark to bright by this time. Nevertheless, we can feel that the hue change of channel V only complete half and we need to get a texture as gray pencil texture with a clear light and shade contrast. While processing channel V can only meet the demand of dark hue change, so we need to process channel $\mathrm{S}$ on the vertical variation as showed in Fig. 6 . Then we can get a texture hue closing to the paper if we do that. 


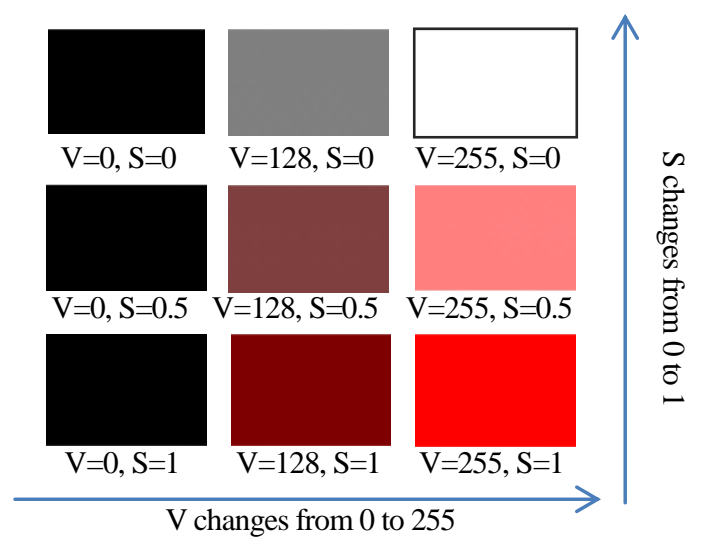

Figure 6. Change of channel V and S

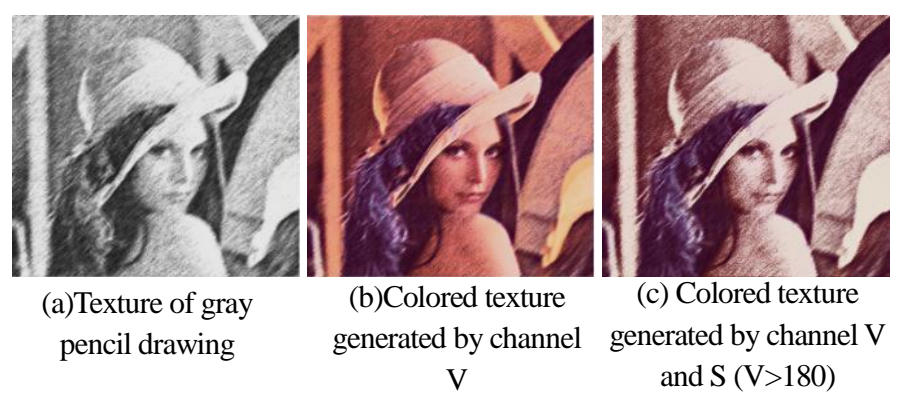

Figure 7. Generation process of colored texture

We plan to get colored texture by virtue of grayscale texture. We need to assign the change rule of grayscale texture to channel $\mathrm{S}$ and $\mathrm{V}$. As show in Fig. 7, the texture value of figure (a) is assigned to channel V in HSV color space and a result is showed in figure (b). But for the result texture, relatively brighter pixels with saturability closing to zero are less because of not processing channel $\mathrm{S}$. As show in figure 7(c), we put the texture value of figure (b) assigned to channel $\mathrm{S}$ only if channel V value is greater than 180 . We assume that grayscale texture value is $\mathrm{G}$ and saturation truncation threshold is $\mathrm{k}$. Then the assignment formula is abbreviated as follows:

$$
\left\{\begin{array}{l}
V=G \\
S=G \text { if } V>k
\end{array}\right.
$$

The range of $\mathrm{k}$ is $0 \sim 255$, while in principle, $\mathrm{k}$ value should be greater than 160 for getting a better effect. Such a set is used to avoid a gray effect as the second image of Fig. 6. At the same time k value should not be greater than 230, otherwise there would not be enough pixels falling into the range $\mathrm{k} \sim 255$. That may give rise to a not obvious saturation stretch effect.

Fig. 8 displays an example of colored texture generated from a 2D image. Brightness and saturability change differently when $\mathrm{k}$ takes different value within $\mathrm{V}>\mathrm{k}$. A comparison from red box textures of (a) and (b) demonstrates that the texture with $\mathrm{V}$ value greater than 200 is more close to the real on account of a assignment process to channel $\mathrm{S}$ within $\mathrm{V}>128$. Another comparison between blue box textures of (b) and (c) proves that the texture presented in picture (b) shows a better light and shade contrast. This is because that the texture of picture (c) within V>230 cannot meet the demand of pixels needed by channel S. 


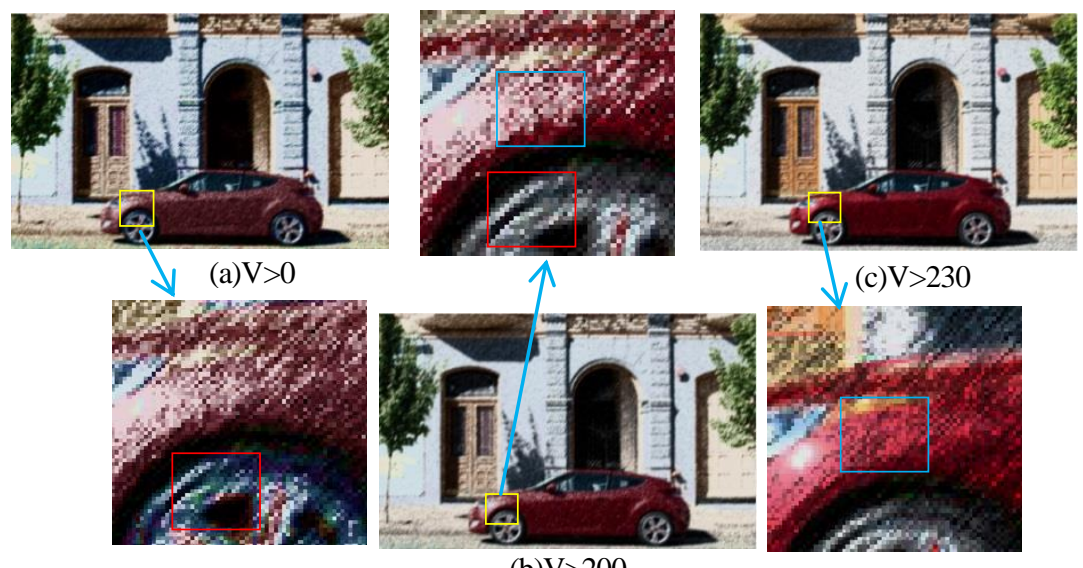

(b) $\mathrm{V}>200$

Figure 8. Texture image with different $\mathrm{V}$

\section{Test Results}

The algorithm in this paper has been realized by $\mathrm{C}++$ language in Android 4.4.2 platform. The hardware device adopted is the mobile phone MI 2S with Adreno (TM) 320@400MHZ. For an image with resolution ratio at $655 * 433$, the outline extraction will take $1.081 \mathrm{~S}$, while the texture rendering takes about 3.231S, with total time consumption for $4.312 \mathrm{~S}$.

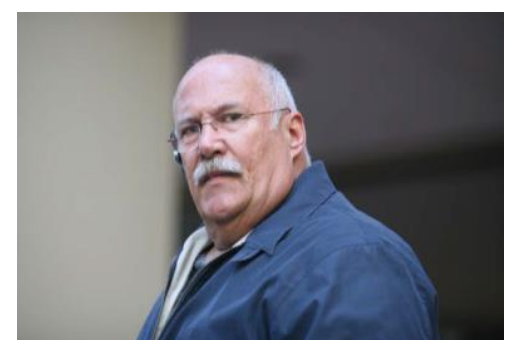

(a)Original image

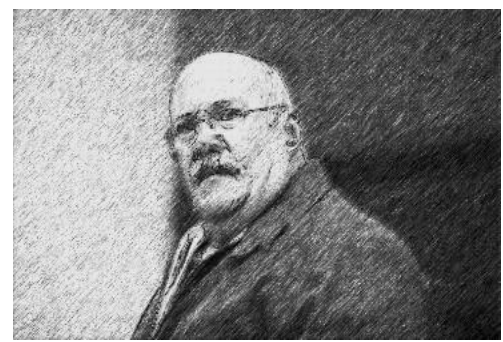

(c) Result for our

gray pencil drawing

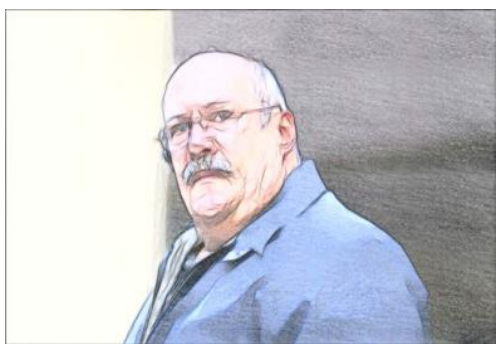

(b) Result for Lu[9]

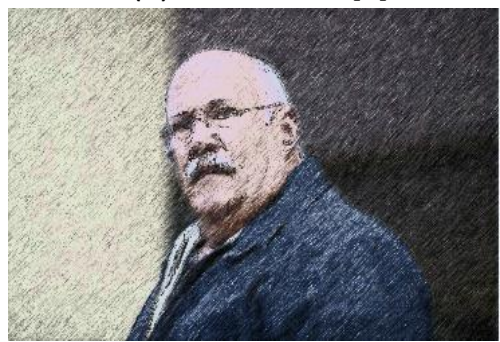

(d) Result for our colored

pencil drawing

Figure 9. Result comparison between $\mathrm{Lu} \mathrm{[9]}$ and our result

In Fig. 9, the test result shows the different simulation conclusions for pencil drawing acquired by utilizing different filtering algorithm. Compared to our approach, a common defect in [1] is a lack of contract especially for losing white paper hue which leads to staying away from the real.

Form the result of our pencil drawing framework, two advantages of our approach can be learned, one is colored texture with more contrast and another is the hue which can better match the original image. Compare to other methods, the colored texture generated by our framework can achieve a good light and shade change and color change. Besides, the final result can better meet the real through improving gray texture contrast and weighting arctangent hue differentiation model (arc tan) to texture. 


\section{Conclusion and Outlook}

In this paper, we propose a colored pencil drawing generation framework through analyzing colored texture by HSV color space and hue differentiation model. We get a better result by it. The experimental result shows that the pencil drawing with colored texture generated by HSV color space and the Arctangent Hue Differentiation Model (arc tan) is more real. But the framework is also defective, because there is no a good conclusion for $\mathrm{k}$ value used as the interceptive value of channel V in HSV when we assign gray texture value to channel S. So that is the point we will make effort for.

\section{References}

[1] C. Lu, L. Xu and J. Jia: Proceedings-the Symposiumon Non-Photorealistic Animation and Rendering (NPAR). (Annecy, France, June .4-6, 2012).p. 65-73.

[2] I. Hutton-jamieson: Colored Pencil Drawing Techniques (North Light Books, USA 1986).

[3] S. Takagi: Pacific Graphics'99 Conference Proceedings (Los Angeles, USA 1999). p. 250-258.

[4] M. K. Yang: A Fast Filter for Colored Pencil Style Drawing.(MS., National Chiao Tung University, China 2006), p.34.

[5] S. Yamamoto, X. Mao and A. Imamiya: Proc. of Pacific Graphics'04 (Seoul, Korea, October.6-9, 2004). p. 329-338.

[6] C. S. Haase and G. W. Meyer: ACM Transactions on Graphics (TOG), Vol. 11 (1992) No.4, p. 305-335.

[7] H. Kang, S. Lee, C. Chui: IEEE Transactions on Visualization and Computer Graphics, Vol.15 (2009) No.1, p.62-76.

[8] D. E. Xie, Y. G. Xuan and Z. L. Zhang: International Conference on Computing, Control and Industrial Engineering (Wuhan, China, June.5-6, 2010). Vol. 2, p. 166-169.

[9] D. E. Xie, H. N. Hu and Z. L. Zhang: Advanced Materials Research, Vols. 433-440 (2012), p.1555-1560.

[10]D. L. Way, M. K. Yang, Z. C. Shih and R. R. Lee: International Journal of Innovative Computing, Information and Control, Vol. 10 (2014) No. 1, p.233-241.

[11]H. J. Lin and Y. H. Li: International Conference on IT Convergence and Security (ICITCS) (Macao, China, December.16-18, 2013). p. 1-4. 